\title{
1 NOXA expression drives synthetic lethality to RUNX1 inhibition in pancreatic cancer
}

2 Josefina Doffo ${ }^{1,2}$, Stefanos A. Bamopoulos ${ }^{1}$, Hazal Köse ${ }^{1}$, Felix Orben ${ }^{3}$, Chuanbing Zang ${ }^{1}$,

3 Miriam Pons ${ }^{4}$, Alexander T. den Dekker ${ }^{5}$, Rutger W. W. Brouwer ${ }^{5}$, Apoorva Baluapuri ${ }^{6}$, Stefan

4 Habringer $^{1}$, Maximillian Reichert ${ }^{3}$, Anuradha Illendula ${ }^{7}$, Oliver H. Krämer ${ }^{4}$, Markus Schick ${ }^{1}$,

5 Elmar Wolf ${ }^{6}$, Wilfred F. J. van IJcken ${ }^{5}$, Irene Esposito ${ }^{8}$, Ulrich Keller ${ }^{1,9,10}$, Günter Schneider ${ }^{3}$,

$6 \quad 9,11$ and Matthias Wirth ${ }^{1}$

$7 \quad{ }^{1}$ Department of Hematology, Oncology and Cancer Immunology, Campus Benjamin Franklin,

8 Charité - Universitätsmedizin Berlin, corporate member of Freie Universität Berlin and

9 Humboldt-Universität zu Berlin, Berlin, Germany

10 '2Stowers Institute for Medical Research, Kansas City, MO, USA

$11{ }^{3}$ Department of Medicine II (Gastroenterology and GI Oncology), Klinikum rechts der Isar,

12 Technische Universität München, School of Medicine, München, Germany

$13{ }^{4}$ Department of Toxicology, University Medical Center, Mainz, Germany

$14{ }^{5}$ Center for Biomics and Department of Cell Biology, Erasmus University Medical Center, 15 Rotterdam, The Netherlands

$16{ }^{6}$ Cancer Systems Biology Group, Department of Biochemistry and Molecular Biology, 17 Biocenter, University of Würzburg, Würzburg, Germany

$18{ }^{7}$ Department of Pharmacology, University of Virginia, Charlottesville, VA, USA

19 Institute of Pathology, Heinrich Heine University and University Hospital of Düsseldorf, 20 Düsseldorf, Germany.

$21{ }^{9}$ German Cancer Research Center (DKFZ) and German Cancer Consortium (DKTK), 22 Heidelberg, Germany 
$24{ }^{11}$ Department of General, Visceral and Pediatric Surgery, University Medical Center Göttingen, 25 Göttingen, Germany

\section{Corresponding author:}

27 Matthias Wirth, Department of Hematology, Oncology and Cancer Immunology, Campus

28 Benjamin Franklin, Charité - Universitätsmedizin Berlin, Hindenburgdamm 30, 12203 Berlin,

29 Germany, e-mail: matthias.wirth@charite.de

30 Keywords: NOXA / Apoptosis / PDAC / RUNX1 / pancreatic cancer 


\section{Significance}

Recent evidence demonstrated the existence of molecular subtypes in pancreatic ductal adenocarcinoma (PDAC), which resist all current therapies. The paucity of therapeutic options, including a complete lack of targeted therapies, underscore the urgent and unmet medical need for the identification of targets and novel treatment strategies for PDAC. Our study unravels a function of the transcription factor RUNX1 in apoptosis regulation in PDAC. We show that pharmacological RUNX1 inhibition in PDAC is feasible and leads to NOXAdependent apoptosis. The development of targeted therapies that influence the transcriptional landscape of PDAC might have great benefits for patients who are resistant to conventional therapies. RUNX1 Inhibition as a new therapeutic intervention offers an attractive strategy for future therapies.

\section{Abstract}

Evasion from drug-induced apoptosis is a crucial mechanism of cancer treatment resistance. The pro-apoptotic protein NOXA marks an aggressive pancreatic ductal adenocarcinoma (PDAC) subtype. To identify drugs that unleash the death-inducing potential of NOXA, we performed an unbiased drug screening experiment. In NOXA-deficient isogenic cellular models we identified an inhibitor of the transcription factor heterodimer $C B F \beta / R U N X 1$. By genetic gain and loss of function experiments we validated that the mode of action depends on RUNX1 and NOXA. Of note, RUNX1 expression is significantly higher in PDACs compared to normal pancreas. We show that pharmacological RUNX1 inhibition significantly blocks tumor growth in vivo and in primary patient-derived PDAC organoids. Through genome wide analysis, we detected that RUNX1-loss reshapes the epigenetic landscape, which gains H3K27ac enrichment at the NOXA promoter. Our study demonstrates a previously unknown mechanism of NOXA-dependent cell death, which can be triggered pharmaceutically. Therefore, our data show a novel way to target a therapy resistant PDAC, an unmet clinical need. 


\section{Introduction}

Pancreatic ductal adenocarcinoma (PDAC) is an aggressive disease often diagnosed at an advanced stage. The incidence of PDAC is steadily increasing, and PDAC is predicted to be the second leading cause of cancer-related death by 2030 (1). Evasion of apoptosis is a characteristic of PDAC and is often associated with treatment resistance (2, 3). A dysregulated transcription commonly results in apoptosis resistance (4). Therefore, the identification of novel concepts to reactivate apoptosis by disrupting cancerous transcription programs is a promising approach for the effective elimination of PDAC cells $(3,5)$.

Comprehensive integrated genome analyses from RNA expression profiles in recent years revealed different subtypes of PDAC with variable biology and therapeutic responsiveness (69). NOXA (latin for damage; also known as PMAIP1 - Phorbol-12-myristate-13-acetateinduced protein 1) is part of an identifier gene-set for the quasi-mesenchymal subtype of the disease (8). This subtype overlaps with the described basal-like and squamous subtype of the disease, which is particularly resistant to the currently used chemotherapeutics $(7,9)$. NOXA belongs to the BCL-2 homology $(\mathrm{BH}) \mathrm{BH}$-only subgroup of the B-cell lymphoma 2 (BCL2) protein family which is essential for the regulation of cell intrinsic apoptosis (10). BCL2 proteins are divided into sensor, effector and protector proteins (10). The classical antiapoptotic protector proteins including Myeloid cell leukemia 1 (MCL1) inhibit effector proteins (e.g. Bcl-2-associated $X$ protein) thereby blocking apoptosis. Pro-apoptotic sensor proteins including NOXA, which directly binds to MCL1, neutralize the anti-apoptotic function of the protector proteins (10), leading to the initiation of apoptotic cell death. In PDAC, NOXA is tightly regulated at the transcriptional level, and transcriptional activation of NOXA by HDACinhibitors or proteasome inhibitors contribute to induce the cell intrinsic apoptosis pathway (11-

81 13). Furthermore, NOXA is regulated by multiple transcription factors including p53 and is involved in apoptosis under genotoxic stress $(14,15)$.

83 Runt-related (RUNX) proteins are master regulators involved in a broad range of biological processes including proliferation, differentiation and apoptosis (16). DNA binding of these 


\section{Materials and Methods}

\section{Cell culture, cell viability assay}

transcription factors is mediated by heterodimerization of a core DNA binding factor alpha chain (CBF $\alpha$ ), composed of one of the three RUNX family members, RUNX1-3, to the nonDNA binding core binding factor beta $(\mathrm{CBF} \beta)$. Each of the three RUNX family members play important roles in different stages of tumor development (17). As has been shown in mouse models, knockouts of any of the three RUNX transcription factors exhibit significant developmental defects: RUNX1 plays an important role in hematopoiesis (18), RUNX2 in bone development (19) and RUNX3 in the gastrointestinal tract (20) and in neurogenesis (21). RUNX expression patterns are highly dynamic and depend on the stage of differentiation, development and environmental conditions (22). In addition, RUNX transcription factors are expressed in almost all cancers (23). Besides its implication in leukemogenesis (24), RUNX1 is strongly expressed in a broad spectrum of solid tumors (25) and is associated with poor prognosis in PDAC (26). Depending on the cellular context, RUNX1 can act both oncogenic and tumor suppressive in solid tumors (27). RUNX1 interacts with various co-factors to shape gene expression. RUNX1-dependent activation of target genes is mediated through an interaction with CBP/p300 (28) and the protein arginine methyltransferase 1 (PRMT1) (29). The inhibitory function of RUNX1 is achieved by interaction with co-repressors such as the Sin3A-HDAC corepressor complex (30).

In this study, we aimed at identifying novel strategies affecting the delicate balance of NOXA expression to drive cell death in an aggressive subtype of PDAC. We found that inhibition of RUNX1 led to a global gain of H3K27ac enrichment contributing to the activation of the proximal NOXA promoter region and suggest a strategy to overcome treatment resistance in an aggressive subtype of PDAC with inferior prognosis.

Cell lines were cultured in Dulbecco's Modified Eagle's Medium (Thermo Fisher Scientific, \#41965062) or Roswell Park Memorial Institute (RPMI) 1640 Medium (Thermo Fisher Scientific, \#21875091) supplemented with 10\% FBS (Thermo Fisher Scientific, \#10270106) 
and 1\% Penicillin/Streptomycin (Thermo Fisher Scientific, \#15070063). They were passaged up to 14 times in a 1:10 dilution every 3-4 days. Murine PDAC cell lines were generated from Kras $^{G 12 D}$ driven mouse models as described (13). For all cell lines used, PCR-based mycoplasma tests were performed at regular intervals. Cell viability was measured by MTTTest (Sigma-Aldrich, \#M5655). Detailed information on the procedures cell viability, drug screening and colony formation assay in Supplementary Materials and Methods.

\section{Patient-derived organoids}

PDAC biopsies and tissues were received from endoscopy punctures or surgical resection. 3D organoids were collected, propagated, and analyzed in agreement with the declaration of Helsinki. This study was approved by the ethical committee of TUM (Project 207/15). Written informed consent from the patients for research use of tumor material was obtained prior to the use. Detailed information in Supplementary Materials and Methods.

Xenograft assays were performed by EPO (Experimental Pharmacology and Oncology, Berlin-

Buch). All animal experiments were approved by the local responsible authorities and

131 Supplementary Materials and Methods.

Statistical Analysis, qChIP, ChIPseq, RNAseq, ATAC seq, 4C, qPCR, Western blot,

135 Detailed information on the procedures and data analyses in Supplementary Materials and 


\section{Results}

High NOXA mRNA expression is associated with an aggressive PDAC subtype

140

141

142

143

144

145

146

147

148

149

150

151

152

153

154

155

156

157

158

159

160

As a stress response, tumor cells may express pro-apoptotic effectors that can be neutralized by anti-apoptotic counterparts, thus dampening the apoptotic response. Such tumor cells expose apoptotic potential. To investigate, if classical pro-apoptotic BH3-only proteins may contribute to this phenotype, we analyzed the mRNA expression of $\mathrm{BH}$-only genes (6) and filtered for transcriptome profiles of human PDAC tumors (Fig. 1A). From this data, we extracted indicated classical $\mathrm{BH} 3$-only genes, performed a hierarchical clustering based on the mRNA expression of the BH3-only genes and subdivided PDAC patient samples into the squamous subtype and combined the ADEX-, classical- and the immunogenic subtypes as "other" subtypes. We subsequently determined if the mRNAs of the listed classical BH3-only members are significantly enriched in these two groups. Of note, specifically high expression of NOXA was significantly $(\mathrm{p}<0.001)$ associated with the squamous subtype (Fig. $1 \mathrm{~A}$ and SFig1A), which is in line with the described expression in quasi-mesenchymal (QM) cancers (8). In accordance with this finding, high NOXA expression ( $>75^{\text {th }}$ percentile) characterized a PDAC patient cohort with inferior survival as compared to patients with low NOXA expression $\left(<25^{\text {th }}\right.$ percentile) in ICGC/Bailey et al. (Fig. 1B and SFig1B) and TCGA datasets (SFig. 1C). We hypothesized that the high expression of NOXA indicates that these tumors harbor an apoptotic potential, and that shifting the balance of apoptosis regulators to a pro-apoptotic state may constitute a therapeutic strategy.

\section{Identification of a synthetic lethal interaction of NOXA and inhibition of RUNX1}

By analyzing transcriptome profiles, we selected human PDAC cell lines of the quasimesenchymal subtype (8) (SFig 1D) and Kras ${ }^{\mathrm{G} 12 \mathrm{D}}$-driven murine PDAC cell lines that exhibit relatively high basal Noxa mRNA expression (SFig. 1E) for cross-species validation to identify drugs that affect the apoptotic balance. To investigate vulnerabilities specifically created by NOXA expression, we generated human and murine isogenic PDAC cell line models with genetically defined NOXA status (SFig. 2A-D). To prove NOXA deficiency, we performed 
western blotting of the two human PDAC cell lines PSN1 and MiaPaCa-2. NOXA protein was absent in $N O X A^{k o}$ cell lines (Fig. 1C). Importantly, NOXA deficiency did not influence the proliferation of the isogeneic cell lines (Fig. 1D).

To identify vulnerabilities associated with NOXA, we next performed a drug screening with a total of 1842 compounds in NOXA-proficient (parental) and NOXA-deficient $\left(N O X A^{k o}\right)$ cells and measured viability (Fig. 1E). Drug testing and viability assays were performed with a single concentration of $600 \mathrm{nM}$, as previously described (13). For identification of effects due of the NOXA status, we used a cut-off of $10 \%$ difference in viability (Fig. 1F). Out of the 1842 compounds we identified 50 drugs that showed higher efficiency in parental cell lines compared to $N O X A^{k o}$ cell lines. Importantly, within the hits found, we identified topoisomerase and proteasome inhibitors, which is in line with data from previous studies (11-13), underlining the robustness of our screening experiment (STable 1). From our screening hits, we selected for specific targeted molecules (target specificity) and novelty (Fig. 1E) and further validated 12 hits with multi-dose experiments (Fig. 1F). Only one of these twelve compounds analyzed in the validation experiments, $\mathrm{Al}-10-49$, showed no growth inhibition in NOX $A^{k o}$ cell lines whereas viability of NOXA-proficient cells was dose-dependently reduced (Fig. 1F). Al-10-49 was

originally designed to inhibit the interaction of the oncofusion protein $\mathrm{CBF} \beta-\mathrm{SMMHC}$ with

RUNX1 (32). The lead compound of the bivalent Al-10-49 has been shown to inhibit as RUNX1/CBF $\beta$ inhibitor in MiaPaCa-2 cells (SFig. 2E). Additionally, using the Swiss TargetPrediction tool (34), CBF $\beta$ was a predicted target of this compound (STable 2). In summary, our data suggest that impairment of RUNX1 activity may affect NOXA-dependent execution of cell death in PDAC.

\section{Induction of NOXA by CBF $\beta / R U N X 1$ inhibition}

191 To investigate whether the Al-10-49-induced drop in viability is mediated by induction of the apoptotic process, we performed fluorometric analysis of Annexin V/PI stained PDAC cells. 
194

associated with a significant induction of cell death in parental cells, whereas only marginal apoptosis induction was observed in NOX $A^{k o}$ cells (Fig. 1G).

We next investigated whether an altered expression of other BCL2 family members such as MCL1, BCL2, BCLXL, BIM, BID, BAK or BAX mediates Al-10-49-induced apoptosis. We detected PARP cleavage but did not detect differential expression of these BCL2 family proteins 6 hours and 24 hours after Al-10-49 treatment (Fig. 1H, SFig. 3A, B), a marked induction of NOXA protein (Fig. 1I) and NOXA mRNA expression (Fig. 1I) was observed upon treatment with Al-10-49. In HCT116 cells harboring wild-type p53, a DNA damage stimulus induced both RUNX1 and p53 and activated p53 target genes, including NOXA (35). To test if p53 is involved in Al-10-49 induced NOXA expression we treated murine cell lines harboring either wiltype, mutant or deleted p53- (SFig. 3C) with Al-10-49 and the topoisomerase II inhibitor etoposide (SFig. 3D). While Noxa induction was highest in wildtype p53 cells upon etoposide treatment, we observed a rather uniform induction of Noxa in each of these cell lines after Al-10-49 treatment (SFig. 3D). Since p53 is mutated in the human PDAC cell lines PSN1 and MiaPaCa-2 we also analyzed the expression of the p53 family member p63, but did not detect significant regulation (SFig. 3E). Both p63 and mutant p53 showed no difference in expression at early time points after treatment with $\mathrm{Al}-10-49$, but tended to show decreased expression after $48 \mathrm{~h}$ and $72 \mathrm{~h}$, respectively (SFig. 3B,E). Our data show that both p53 ${ }^{\mathrm{mut}}$ and RUNX1 are not induced upon Al-10-49 treatment (SFig. 3B, E). Contrary, we rather observed a decreased expression of RUNX1 after Al-10-49 treatment in MiaPaCa-2 and PSN1 cells (SFig. 3E).

Next, to substantiate caspase-induced apoptosis, we examined Al-10-49 together with the pan-caspase inhibitor zVAD-FMK by Annexin V/PI FACS. Here, we observed a significant rescue in apoptosis induction (Annexin $\mathrm{V}^{+} / \mathrm{PI}^{-}$fraction) (SFigure 3F). Both fractions, i.e. Annexin $\mathrm{V}^{+} / \mathrm{PI}^{-}$and Annexin $\mathrm{V}^{+} / \mathrm{PI}^{+}$, remained unaffected in $N O X A^{k o}$ cells, indicating the relevance of NOXA in cell death (SFigure 3F).

To further investigate the role of NOXA in Al-10-49 induced cell death, we generated MiaPaCa2 cells stably expressing the CRISPR activator (CRISPRa) dCas9-MS2-p65-HSF1 (MpH), and 
an established NOXA sgRNA to endogenously overexpress NOXA (SFig. 2E, SFig. 3G). NOXA-CRISPRa cells phenocopied Al-10-49 treated cells in clonogenic assays and, more (SFig. 3H, I).

Together, our data show that inhibition of RUNX1 by Al-10-49 induces NOXA mRNA and protein expression and thereby drives NOXA-dependent apoptosis.

\section{RUNX1 is upregulated in pancreatic cancer and suppresses NOXA expression}

230

231

232

233

234

235

236

237

238

239

240

241

242

Understanding the mode of action of drugs is critical for the implementation of patient stratification strategies. To address this question, we next investigated how Al-10-49 induces NOXA expression. Since AI-10-49 inhibits the interaction between $\mathrm{CBF} \beta$ and a DNA binding $\alpha$ subunit encoded by RUNX proteins (16), we tested whether loss of RUNX expression affects NOXA expression in knockouts of all three RUNX genes $R U N X 1, R U N X 2$ and $R U N X 3$ in MiaPaCa-2 cells (SFig 2F). Remarkably, we observed an induction of NOXA mRNA solely in RUNX1 knockout cells (Fig. 2A), arguing for a RUNX1-specific repression of the NOXA gene.

We validated this effect by siRNA-mediated RUNX1 depletion in Panc1, AsPC1 and MiaPaCa2 cells (SFig. 4A). In addition to the induction of NOXA mRNA, we also observed a significant NOXA induction in $R U N X 1^{k o}$ cells at the protein level (Fig. 2B). To analyze whether $R U N X 1^{k o}$ cells have a growth disadvantage, we compared colony formation of $R U N X 1^{\text {ko }}$ cells to the parental cell line. Here, we observed significantly reduced formation of colonies (Fig. 2C), which phenocopied the effects observed in Al-10-49 treated cells (SFig. $3 \mathrm{H}, \mathrm{I}$ ). In addition, $R U N X 1^{k o}$ cells showed an increased cell death rate at basal levels, as demonstrated by Annexin V flow cytometry (Fig 2D). To demonstrate the specificity of Al-10-49 in this context, we applied Al-10-49 in two RUNX1 ${ }^{k o}$ clones as well as in RUNX1 siRNA-treated MiaPaCa-2 cells. Although we did not observe a consistent effect in the RUNX1 knockout clones, which is probably related to the difficult cultivation of these cells, the use of siRNA, on the other hand, showed reproducible effects equivalent to a doubling of the $\mathrm{GI}_{50}$ value, confirming the 
dependence on RUNX1 (SFig. 4B). These genetic experiments identify RUNX1 as negative regulator of NOXA gene expression.

251 To further investigate the relevance of RUNX1 in PDAC, we analyzed RUNX1 expression in human and murine datasets. We found that RUNX1 indeed is transcriptionally upregulated in pancreatic cancer in all three datasets investigated (Fig. 2E, SFig4C). In addition, RUNX1 was expressed in pancreatic cancer, whereas it was not detected in normal pancreas on protein level (Fig. 2F). In premalignant Kras ${ }^{\mathrm{G} 12 \mathrm{D}}$ driven murine pancreatic epithelial cells Runx1 mRNA (Fig. 2G) and RUNX1 target gene signatures (Fig. 2H) were significantly enriched compared to normal pancreatic epithelial cells, displaying specificity for RUNX1 expression in nonstromal tumor-initiating cells. During PDAC progression RUNX1 expression is maintained. Suspecting a RUNX1-mediated repression of the NOXA gene, we re-analyzed the ICGC/Bailey et al. (6) and Collisson et al. (8) transcriptome datasets and indeed observed a negative correlation trend of RUNX1 and NOXA expression in the squamous/quasimesenchymal PDAC subtype (SFig. 4D). To investigate whether RUNX1 expression was the main contributing factor to NOXA expression, we performed a shRNA-mediated knockdown of NOXA in RUNX $1^{k o}$ cells (SFig. 4E). Congruent with our initial findings, the basal apoptosis rates were restored (SFig. $4 \mathrm{~F})$.

Taken together, these data argue for a RUNX1-mediated repression of NOXA expression.

\section{RUNX1 inhibition induces NOXA through amplification of active chromatin marks}

To understand the immediate effect of Al-10-49 treatment, we performed RNAseq analyses of MiaPaCa2 cells $6 \mathrm{~h}$ upon treatment in comparison to vehicle treated cells. Apart from NOXA, which was upregulated in treated cells, less than 300 genes, were found to be differentially expressed, suggesting a high specificity of Al-10-49 (Fig. 3A). Gene set enrichment analyses

273 revealed a significant apoptosis signature and a negative enrichment score for RUNX1 targets 274 (Fig. 3B, STable 3) upon Al-10-49 treatment. To analyze the impact of RUNX1 inhibition on 275 chromatin accessibility we performed assays for transposase accessible chromatin with high276 throughput sequencing (ATAC-seq). We observed reduced chromatin accessibility after Al-10- 
27749 treatment, compared to the vehicle control $6 \mathrm{~h}$ after Al-10-49 treatment (Fig. 3C). In addition,

278 a comparison of parental MiaPaCa-2 cells with isogenic RUNX1 deficient cells showed a an

279 increased accessibility of the chromatin.

280 To investigate the association of RUNX1 inhibition with regulation of chromatin dynamics, we performed H3K27ac ChIPseq experiments to detect transcriptionally active chromatin. A crosscoverage and fingerprint plot showed adequate signal strength in enriched regions (SFig. 5A, B). Globally, H3K27ac signal was increased in both replicates (Fig. 3D, SFig. 5C), arguing for a neutralization of the repressor activity of RUNX1.

We also performed RUNX1 ChIPseq to assess the impact of RUNX1 inhibition on RUNX1 binding. This analysis showed a peak downstream of the NOXA gene, which we hypothesized to be an enhancer region and coined it downstream binding site 1 (dBS1). To substantiate our findings, we analyzed RUNX1 ChIPseq from K562 and MCF10A cells. Indeed, we found an overlap of our identified peak in both ChIPseq datasets and were thus able to validate the peak identified in MiaPaCa-2 cells (SFig. 6A). In both, ChIPseq and qChIP experiments, we observed a drop in RUNX1 binding at downstream binding site 1 (dBS1) and an increased acetylation of $\mathrm{H} 3 \mathrm{~K} 27$ at the NOXA promoter upon Al-10-49 treatment (Fig. 3E, F), indicating an activation of the gene by acetylation of the proximal promoter region of NOXA, subsequently leading to increased gene expression. To analyze the spatial organization of the NOXA region, we performed chromosome conformation capture assays (4C) to capture interactions between the NOXA locus (view point) and all other genomic loci. Here, we found a hitherto unknown interaction with a downstream region of the NOXA gene, which is abrogated upon Al-10-49 treatment and in $R U N X 1^{k o}$ cells (Fig. 3E, arrow/dBS1, SFig. 6A). Binding of the nuclear protein CCCTC-binding factor (CTCF), which marks insulator regions to prevent crosstalk between active and inactive chromatin, was unaffected (Fig. 3E). Additionally, RUNX1 peaks in the vehicle control (indicated by an arrow in Fig. 3E) and at the NOXA gene, arguing for a spatial 302 interaction, which is mediated by RUNX1 (Fig. 3E, G). The dBS1 region was the only region 303 within the CTCF boundaries where both RUNX1 binding and DNA-DNA interaction had 304 disappeared after Al-10-49 (Fig. 3G, SFig. 6A). This spatial interaction could also be found in 
public Hi-C data of Panc1, K562 cells, and to a lower extend in the epithelial cell line MCF10A (Fig. 3F, SFig. 6B) (36). Taken together, these data suggest that RUNX1 binding to the dBS1 region actively represses the NOXA gene. To identify which histone deacetylases are responsible for this effect, we used class I HDAC inhibitors. Here, in particular, inhibition of HDAC1/2 by Merck60 showed a significant induction of NOXA mRNA expression (SFig. 6C) as well as an induction of the H3K27ac mark at the NOXA promoter (SFig. 6D). Additionally, murine PDAC cells harboring a dual recombinase system and a 4-hydroxy-tamoxifen inducible Cre to knockout alleles for either Hdac1 (PPT-F3641) or Hdac2 (PPT-F1648) (SFig. 6E), displayed a Noxa induction only in Hdac2 deleted cells (SFig. 6F), which is in line with a previous study showing that HDAC2 is responsible for the repression of NOXA in PDAC (11). Therefore, a HDAC2-RUNX1/CBF $\beta$ axis might be responsible for NOXA repression. This requires further validation.

To prove that the $d B S 1$ region is causative for the repression of NOXA, we first screened this

318 region for evolutionary conserved $R U N X 1$ binding motifs, and indeed identified conserved 319 RUNX1 consensus sequences (SFig.6G). We next performed a CRISPR/Cas9-mediated 320 knockout of the $d B S 1$ region (SFig. $6 \mathrm{H}$ ) to genetically demonstarte its involvement in NOXA 321 repression. Indeed, loss of the $d B S 1$ region led to increased NOXA mRNA (Fig. $3 \mathrm{H}$ ) and protein levels (Fig. 3I). This demonstrates the repressive function of this RUNX1 binding site. In contrast to parental cells, Al-10-49 treatment did not further affected NOXA expression (Fig. treatment, albeit to a lesser extent (Fig. 3H, I).

326 In summary, we describe a previously unknown mechanism of a RUNX1-mediated repression 327 of the NOXA gene in PDAC. Through enrichment of an active chromatin mark at the NOXA 328 gene itself, its expression is significantly increased (Fig. 3J), thereby inducing apoptosis. This 329 mechanism could be crucial for therapeutic interventions that depend on a NOXA-induced cell 330 death program. 
332 To validate whether RUNX1 inhibition could be effective in vivo, we first examined the efficacy 333 of Al-10-49 in mice carrying MiaPaCa-2 PDAC xenografts (Fig. 4A). Al-10-49 treatment 334 resulted in a significant decrease in tumor volume (Fig. 4B) and proliferative capacity (Ki67, 335 Fig. 4C). Importantly, Al-10-49 treatment induced apoptosis measured by cleaved caspase 3 336 (CC3) positivity in the tumor compared to the vehicle control in parental cells (Fig. 4B, C). 337 Whereas parental cells did not change in tumor volume after Al-10-49 treatment, NOXA ${ }^{k o}$ cells 338 still grew upon treatment, supporting NOXA as an essential contributor of Al-10-49 efficacy 339 (Fig. 4B). Additionally, no significant difference between control and treatment was observed 340 in either K67 or CC3 stainings in $N O X A^{k o}$ cells (Fig. 4D). Taken together, these data indicate 341 that apoptosis induction by Al-10-49 treatment is also dependent on NOXA in vivo.

342 We next isolated seven human patient-derived organoids (PDOs) from PDAC patients to 343 investigate RUNX1 inhibition by Al-10-49. First, we performed transcriptome profiling (STable 344 4), and sorted PDOs for high and low NOXA mRNA expression (Fig. 4E). Gene set enrichment 345 analysis revealed a significant $(\mathrm{q}<0.001)$ accumulation of an apoptosis signature in the PDOs 346 with a high NOXA expression (Fig. 4F). PDOs with a high NOXA expression showed the 347 strongest growth inhibition towards Al-10-49 treatment, which further supports our previous 348 findings (Fig. 4G).

349 Overall, these findings show that RUNX1 inhibition might be a novel therapeutic option to treat 350 PDAC.

\section{Discussion}

Molecular tumor profiling and functional studies have led to the identification and validation of genes and signaling pathways that are dysregulated or mutated in PDAC (7, 37-39). Based on comprehensive molecular characterization of PDACs (6-9), it might thus be possible to define personalized treatment strategies (38). An imbalance of signaling pathways, such as cell death-associated pathways, promote tumor maintenance and treatment resistance in PDAC

358 (3). In this study, we analyzed publicly available transcriptome profiles of PDAC patients and 
mesenchymal subtype. In contrast to NOXA, which is tightly regulated at the transcriptional level in PDAC (11), one of its anti-apoptotic counterparts, MCL1 (10), is mostly regulated at the protein level (40). Since NOXA mRNA and protein expression do not correlate strongly, as has been shown in mantle cell lymphoma (41) and in PDAC (11), it is important to identify substances that can induce apoptosis by taking advantage of a NOXA-associated vulnerability. We therefore performed drug-screening experiments in isogenic cell models with NOXAdeficient and NOXA-proficient counterparts to search for compounds that can exploit this vulnerability. We unexpectedly found a substance that inhibits the core binding alpha units RUNX1, RUNX2 or RUNX3 with CBF $\beta$.

The functions of RUNX1 are highly specific depending on the tissue and cell type. Deletion of Runx1 in a mouse model of T-cell acute lymphoblastic leukemia (T-ALL)(42), silencing of RUNX1 in human T-ALL cells (42) and silencing of RUNX1 in SW480 human colon cancer cells (43) all triggered apoptosis. In contrast, in Kasumi-1 t(8;21) leukemia cells RUNX1 overexpression induced apoptosis by eliciting expression of the cyclin-dependent kinase inhibitor p57 $7^{\text {Kip2 }}$ (44). In line with the biological effects observed in T-ALL (42) and in colon cancer cells (43), we observed an induction of apoptosis both through the pharmacological CBF $\beta / R U N X 1$ inhibition by the compound AI-10-49 and in CRISPR/Cas9 mediated knockouts of RUNX1. We observed a significant induction of NOXA mRNA expression, which was exclusive to RUNX1 knockout cells and could not be observed in RUNX2 or RUNX3 knockout cells, arguing for a non-redundant function for RUNX1.

In a chemical high-throughput screen, which was performed to identify compounds that disrupt the interaction between RUNX1 and the CBF $\beta$-MYH11/SMMHC fusion protein first a lead molecule was identified, which exhibited low selectivity for the fusion protein (33). Therefore, a bivalent derivate of this compound was generated. Al-10-49 inhibits CBF $\beta$-MYH11/SMMHC with an increased selectivity and restores the formation of wild-type CBF $\beta$-RUNX1 (33). In cells, lacking the $\mathrm{CBF} \beta-\mathrm{MYH} 11 / \mathrm{SMMHC}$ fusion protein, $\mathrm{Al}-10-49$ acts like the monomeric lead molecule and inhibits wild-type $\mathrm{CBF} \beta / \mathrm{RUNX1}$. In fact, both Al-10-49 treatment as a putative 
unexpectedly showed an induction of NOXA, similarities in transcriptional regulation at a genome-wide scale and an associated apoptosis induction in PDAC cells, arguing for RUNX1 as a repressor of NOXA gene expression. We could show that the pharmacological CBF $\beta / R U N X 1$ inhibition leads to a global enrichment of H3K27ac, a marker for active chromatin, including the NOXA gene. We observed an unexpected punctual interaction of a NOXA downstream RUNX1 binding site and the NOXA promoter. How exactly RUNX1 exercises its repressive function in PDAC has to be addressed in detail in further studies. An analysis of different data sets showed a high expression of RUNX1 in pancreatic cancer compared to normal pancreas. Of note, knockdown of $R U N X 1$ in PDAC cells was shown to suppress the invasive/aggressive phenotype via regulation of miR-93 (45). In particular, high RUNX1 expression and RUNX1 target gene signatures were observed in premalignant $\mathrm{Kras}^{\mathrm{G} 12 \mathrm{D}}$ driven murine pancreatic epithelial cells, which together indicate a largely unexplored and possibly unexploited relevance of RUNX1 in PDAC.

401 Since the apoptosis machinery in PDAC cells retains its functionality (3), the strategy of directly 402 inhibiting pro-survival BCL2 proteins such as the NOXA antagonist MCL1 appears extremely 403 attractive (46). One of the first selective MCL1 inhibitors with in vivo activity, S63845, showed massive apoptosis induction in multiple myeloma and acute myeloid leukemia, but many solid tumors were resistant to $\mathbf{S} 63845$ monotherapy (47). Combination therapies such as $\mathbf{S 6 3 8 4 5}$ with the SRC kinase inhibitor Dasatinib reduced cell viability in PDAC models and even lead to a reduction in metastasis formation (48). This data shows that the development of new 408 therapeutic strategies, especially with regard to apoptosis evasion for PDAC, are extremely 409 promising in improving the current clinical regimens. Whether compounds like S63845 synergistically combine with $\mathrm{Al}-10-49$ is currently under investigation.

411 Unraveling the transcriptional regulation of apoptosis-associated genes and the interplay of 412 transcription factors, such as RUNX1, is important to understand the tumor biology of PDAC. 413 The development and improvement of compounds that can inhibit transcription factors, such 414 as the CBF $\beta / R U N X 1$ inhibitor used here, perhaps in combination with proteolysis-targeting chimeras (PROTAC) technology (49), could provide new approaches for PDAC treatment. 
416 Therefore, our mechanistic work, demonstrating a control of NOXA by a repressive facet of

417 RUNX1 opens a novel research direction into potent RUNX1 inhibitors and a novel way to

418 target this deadly disease.

419

420

\section{Acknowledgements}

421 We thank Martin Schlensog and Yakup Yasar for performing the IHC staining. We thank Dieter 422 Saur for providing murine PDAC cell lines and thank Ernesto Acevedo for providing the 423 p53(1C12) antibody. Funding: Else-Kröner-Fresenius Stiftung (2016_A43 to M.W.); Walter 424 Schulz Stiftung to M.W.; Deutsche Forschungsgemeinschaft (DFG) [SFB1321 (Project425 ID 329628492) C13 to G.S. and SCHN 959/6-1 and SCHN 959/3-2 to G.S.] and SFB 1335 426 project P3 to U.K.; Wilhelm-Sander-Stiftung (2017.048.2 to G.S. and U.K.); Deutsche Krebshilfe project 70114425 to U.K.; Stiftung Charité to U.K..

428

429 430

\section{Conflict of Interest}

The authors declare no competing interests.

\section{Availability of Data and Materials}

RNAseq (cell lines), ChIPseq, ATACseq and 4C accession No.: PRJEB39828. RNAseq (organoids) gene expression matrix is shown in the STable 4.

\section{References}

2. D. Hanahan, R. A. Weinberg, Hallmarks of cancer: the next generation. Cell 144, ductal adenocarcinoma. Mol Cancer 7, 64 (2008). 
4414 J. E. Bradner, D. Hnisz, R. A. Young, Transcriptional Addiction in Cancer. Cell 168, 629-643 (2017).

443 5. Y. Cheng et al., Targeting epigenetic regulators for cancer therapy: mechanisms and advances in clinical trials. Signal Transduct Target Ther 4, 62 (2019).

6. P. Bailey et al., Genomic analyses identify molecular subtypes of pancreatic cancer. Nature 531, 47-52 (2016).

447 7. E. A. Collisson, P. Bailey, D. K. Chang, A. V. Biankin, Molecular subtypes of 448 pancreatic cancer. Nat Rev Gastroenterol Hepatol 16, 207-220 (2019).

449 8. E. A. Collisson et al., Subtypes of pancreatic ductal adenocarcinoma and their 450 differing responses to therapy. Nat Med 17, 500-503 (2011).

9. R. A. Moffitt et al., Virtual microdissection identifies distinct tumor- and stroma-specific subtypes of pancreatic ductal adenocarcinoma. Nat Genet 47, 1168-1178 (2015).

10. A. Strasser, S. Cory, J. M. Adams, Deciphering the rules of programmed cell death to improve therapy of cancer and other diseases. EMBO J 30, 3667-3683 (2011).

11. P. Fritsche et al., HDAC2 mediates therapeutic resistance of pancreatic cancer cells via the BH3-only protein NOXA. Gut 58, 1399-1409 (2009).

12. M. Wirth et al., MYC and EGR1 synergize to trigger tumor cell death by controlling NOXA and BIM transcription upon treatment with the proteasome inhibitor bortezomib. Nucleic Acids Res 42, 10433-10447 (2014).

13. K. Lankes et al., Targeting the ubiquitin-proteasome system in a pancreatic cancer subtype with hyperactive MYC. Mol Oncol 14, 3048-3064 (2020).

14. E. Oda et al., Noxa, a BH3-only member of the Bcl-2 family and candidate mediator of p53-induced apoptosis. Science 288, 1053-1058 (2000).

464 15. C. Ploner, R. Kofler, A. Villunger, Noxa: at the tip of the balance between life and 465 death. Oncogene 27 Suppl 1, S84-92 (2008).

466 16. L. S. Chuang, K. Ito, Y. Ito, RUNX family: Regulation and diversification of roles through interacting proteins. Int J Cancer 132, 1260-1271 (2013). 
17. B. A. Otalora-Otalora, B. Henriquez, L. Lopez-Kleine, A. Rojas, RUNX family: Oncogenes or tumor suppressors (Review). Oncol Rep 42, 3-19 (2019).

18. M. Ichikawa et al., AML-1 is required for megakaryocytic maturation and lymphocytic differentiation, but not for maintenance of hematopoietic stem cells in adult hematopoiesis. Nat Med 10, 299-304 (2004).

19. Y. Lou et al., A Runx2 threshold for the cleidocranial dysplasia phenotype. Hum Mol Genet 18, 556-568 (2009).

20. H. Fukamachi, K. Ito, Growth regulation of gastric epithelial cells by Runx3. Oncogene 23, 4330-4335 (2004).

21. K. Inoue et al., Runx3 controls the axonal projection of proprioceptive dorsal root ganglion neurons. Nat Neurosci 5, 946-954 (2002).

22. R. Mevel, J. E. Draper, A. L. M. Lie, V. Kouskoff, G. Lacaud, RUNX transcription factors: orchestrators of development. Development 146 (2019).

23. M. Uhlen et al., A pathology atlas of the human cancer transcriptome. Science $\mathbf{3 5 7}$ (2017).

24. K. Shigesada, B. van de Sluis, P. P. Liu, Mechanism of leukemogenesis by the inv(16) chimeric gene CBFB/PEBP2B-MHY11. Oncogene 23, 4297-4307 (2004).

25. C. J. Scheitz, T. S. Lee, D. J. McDermitt, T. Tumbar, Defining a tissue stem celldriven Runx1/Stat3 signalling axis in epithelial cancer. EMBO J 31, 4124-4139 (2012).

26. D. J. Birnbaum et al., Expression of Genes with Copy Number Alterations and Survival of Patients with Pancreatic Adenocarcinoma. Cancer Genomics Proteomics 13, $191-200$ (2016).

27. C. J. Scheitz, T. Tumbar, New insights into the role of Runx1 in epithelial stem cell biology and pathology. J Cell Biochem 114, 985-993 (2013).

493 28. I. Kitabayashi, A. Yokoyama, K. Shimizu, M. Ohki, Interaction and functional cooperation of the leukemia-associated factors AML1 and p300 in myeloid cell differentiation. EMBO J 17, 2994-3004 (1998). 
29. X. Zhao et al., Methylation of RUNX1 by PRMT1 abrogates SIN3A binding and potentiates its transcriptional activity. Genes Dev 22, 640-653 (2008).

30. Z. Sheng, S. Z. Wang, M. R. Green, Transcription and signalling pathways involved in BCR-ABL-mediated misregulation of 24p3 and 24p3R. EMBO J 28, 866-876 (2009).

31. M. Chan-Seng-Yue et al., Transcription phenotypes of pancreatic cancer are driven by genomic events during tumor evolution. Nat Genet 52, 231-240 (2020).

32. J. A. Pulikkan et al., CBFbeta-SMMHC Inhibition Triggers Apoptosis by Disrupting MYC Chromatin Dynamics in Acute Myeloid Leukemia. Cell 174, 172-186 e121 (2018).

33. A. Illendula et al., Chemical biology. A small-molecule inhibitor of the aberrant transcription factor CBFbeta-SMMHC delays leukemia in mice. Science 347, 779-784 (2015).

34. D. Gfeller et al., SwissTargetPrediction: a web server for target prediction of bioactive small molecules. Nucleic Acids Res 42, W32-38 (2014).

35. D. Wu, T. Ozaki, Y. Yoshihara, N. Kubo, A. Nakagawara, Runt-related transcription factor 1 (RUNX1) stimulates tumor suppressor p53 protein in response to DNA damage through complex formation and acetylation. J Biol Chem 288, 1353-1364 (2013). genome. Nature 489, 57-74 (2012).

37. M. Orth et al., Pancreatic ductal adenocarcinoma: biological hallmarks, current status, and future perspectives of combined modality treatment approaches. Radiat Oncol 14, 141 (2019).

38. C. Torres, P. J. Grippo, Pancreatic cancer subtypes: a roadmap for precision medicine. Ann Med 50, 277-287 (2018).

521 39. A. Biederstadt et al., SUMO pathway inhibition targets an aggressive pancreatic cancer subtype. Gut 69, 1472-1482 (2020). 
40. R. M. Fritsch, G. Schneider, D. Saur, M. Scheibel, R. M. Schmid, Translational repression of MCL-1 couples stress-induced elF2 alpha phosphorylation to mitochondrial apoptosis initiation. J Biol Chem 282, 22551-22562 (2007).

41. M. A. Dengler et al., Discrepant NOXA (PMAIP1) transcript and NOXA protein levels: a potential Achilles' heel in mantle cell lymphoma. Cell Death Dis 5, e1013 (2014).

42. A. Choi et al., RUNX1 is required for oncogenic Myb and Myc enhancer activity in Tcell acute lymphoblastic leukemia. Blood 130, 1722-1733 (2017).

43. Y. Zhou et al., LRG1 promotes proliferation and inhibits apoptosis in colorectal cancer cells via RUNX1 activation. PLoS One 12, e0175122 (2017).

44. S. Liu et al., RUNX1 inhibits proliferation and induces apoptosis of $t(8 ; 21)$ leukemia cells via KLF4-mediated transactivation of P57. Haematologica 104, 1597-1607 (2019).

45. Y. Cheng et al., RUNX1 promote invasiveness in pancreatic ductal adenocarcinoma through regulating miR-93. Oncotarget 8, 99567-99579 (2017).

46. S. H. Wei et al., Inducing apoptosis and enhancing chemosensitivity to gemcitabine via RNA interference targeting Mcl-1 gene in pancreatic carcinoma cell. Cancer Chemother Pharmacol 62, 1055-1064 (2008).

47. A. Kotschy et al., The MCL1 inhibitor S63845 is tolerable and effective in diverse cancer models. Nature 538, 477-482 (2016).

48. L. Castillo et al., MCL-1 antagonism enhances the anti-invasive effects of dasatinib in pancreatic adenocarcinoma. Oncogene 39, 1821-1829 (2020).

544 49. X. Sun et al., PROTACs: great opportunities for academia and industry. Signal Transduct Target Ther 4, 64 (2019).

546 50. C. D. Logsdon et al., Molecular profiling of pancreatic adenocarcinoma and chronic pancreatitis identifies multiple genes differentially regulated in pancreatic cancer. Cancer Res 63, 2649-2657 (2003).

549 51. M. Uhlen et al., Proteomics. Tissue-based map of the human proteome. Science 347, 1260419 (2015). 
52. D. Alonso-Curbelo et al., A gene-environment-induced epigenetic program initiates tumorigenesis. Nature 590, 642-648 (2021).

\section{Figure Legends}

555 Figure 1 Screening for NOXA associated vulnerabilities in PDAC cells

556 A) Cluster analysis of mRNA from classical BH3-only proteins, derived from transcriptome 557 profiles of PDAC patients (6). Molecular subtypes have been divided into two groups: the 558 aggressive squamous subtype and all other subtypes have been merged to others. Histological 559 Subtypes are indicated. Upper and lower quartiles of indicated mRNAs were identified. 560 Significant (Fisher exact test) frequencies of a high mRNA expression of the indicated genes 561 (upper quartile) in the squamous subtype are indicated $\left({ }^{*} p<0.05,{ }^{* *} p<0.01,{ }^{* * *} p<0.001\right)$. B) 562 Survival of PDAC patients with a low (lower quartile) and a high (upper quartile) NOXA mRNA 563 expression, derived from the dataset described in $A)(6)$. Log rank test: $p=0.006$. C) Western 564 blot analysis of NOXA protein in PSN1 and MiaPaCa-2 parental and isogenic NOX ${ }^{\mathrm{ko}}$ cell 565 lines. Vinculin served as loading control. D) Growth curves of PSN1 and MiaPaCa-2 parental 566 and isogenic $N O X A^{k o}$ cell lines performed with live-cell imaging. 5 pictures per well were taken 567 every $8 \mathrm{~h}$ and growth was calculated as confluence (\%) normalized to $0 \mathrm{~h}$ control. E) Schematic representation of the performed high throughput drug screening strategy. 4 human pancreatic cancer cell lines (2 parental and $2 N O X A^{k o}$ ) and 4 murine cell lines (2 parental and 2 NOX $A^{k o}$ ) were used for screening a total of 1842 drugs. These compounds were added to each cell line

$57124 \mathrm{~h}$ after seeding at a concentration of $600 \mathrm{nM}$ and cell viability was measured by MTT assay 572 after 72 h. $\mathrm{n}=3$; all biological replicates were performed as technical triplicates. The inhibitors 573 that differentially reduced viability in parental cell lines up to $10 \%$ more in comparison to $574 N O X A^{k o}$ cells were further followed. Based on target treatment and or/novelty, 12 drugs were selected out of the first 50 hits. The $\mathrm{Gl}_{50}$ of the drugs for murine and human cell lines was

576 calculated from dose-response treatment using MTT assay. F) Dose-response treatment in 8 577 human and murine pancreatic cancer cell lines (4 parental, $4 N O X A^{k o}$ ). The fold change of the 
$578 \mathrm{Gl}_{50}$ of the knockout cell lines compared to the parental is depicted. $\mathrm{n}=3$; all biological replicates

579 were performed as technical triplicates. Red represents sensitivity in parental cell line in 580 respect to its isogenic counterpart (smaller $\mathrm{Gl}_{50}$ ). Blue stands for higher sensitivity in the 581 knockout cell line. $\mathrm{R}=$ resistant cell line within the used doses. Dose-response inhibition was calculated with logarithmic regression and tested for significance with logit model $\left({ }^{*} p<0.05\right.$, $\left.{ }^{* *} \mathrm{p}<0.01,{ }^{* \star *} \mathrm{p}<0.001\right)$ G) FACS analysis of Annexin V/PI stained parental and $N O X A^{k o}$ and

584 cells after $72 \mathrm{~h}$ treatment with $3 \mu \mathrm{M} \mathrm{Al-10-49(++)}$ or DMSO (-) as vehicle control. $\mathrm{n}=3$; all 585 biological replicates were performed as technical triplicates. $p$ value of unpaired t-test $\left.{ }^{* * *} p<0.001 . \mathrm{H}\right)$ Western blot analysis of NOXA and MCL1 proteins in pancreatic cancer cell lines upon $6 \mathrm{~h} \mathrm{Al-10-49} \mathrm{treatment.} \mathrm{Representative} \mathrm{Western} \mathrm{blot} \mathrm{is} \mathrm{shown.} \mathrm{Vinculin} \mathrm{served} \mathrm{as}$ loading control. $n=3$; all biological replicates were performed as technical triplicates. (-) DMSO, (+) $1.5 \mu \mathrm{M} \mathrm{Al}-10-49,(++) 3 \mu \mathrm{M} \mathrm{Al}-10-49$. I) qPCR of NOXA in PSN1 and MiaPaCa-2 cell lines.

590 Conditions as described in $\mathrm{H}$ ).

591 Figure 2 RUNX1 is upregulated in pancreatic cancer and a genetic RUNX1 deletion induces $592 \quad$ NOXA transcription and apoptosis

593 A) qPCR of $N O X A$ in parental, $R U N X 1^{k o}, R U N X 2^{k o}$ and $R U N X 3^{k o}$ MiaPaCa-2 cells. mRNA fold 594 change was calculated in comparison to parental cell line. $n=3$; all biological replicates were 595 performed as technical triplicates. B) Western blot analysis and quantification of NOXA and 596 RUNX1 proteins in parental, $N O X A^{k o}$ and $R U N X 1^{k o}$ MiaPaCa-2 cells. Tubulin and actin served as loading controls. * unspecific band. P value of t-test, $p<0.05 . \mathrm{C})$ Representative image and quantification of clonogenic assay in parental and $R U N X 1^{k o}$ cells. $\mathrm{n}=3$; all biological replicates were performed as technical triplicates. $\mathrm{P}$ value of $\mathrm{t}$-test, $\mathrm{p}<0.05$.

D) FACS analysis of 600 Annexin $\mathrm{V}$ stained parental and $R U N X 1^{k o}$ MiaPaCa-2 cells. $\mathrm{n}=3$; all biological replicates were 601 performed as technical triplicates. $\mathrm{P}$ value of t-test, $\mathrm{p}<0.01$. E) $R U N X 1$ mRNA expression of 602 patient samples from normal pancreas and pancreatic cancer (50), accessed via the 603 oncomine.org, $\mathrm{p}<0.01$. F) IHC of patient samples from normal pancreas and pancreatic cancer accessed via the human protein atlas (51). G) RUNX1 mRNA expression of mouse samples 
605 from normal pancreatic epithelial cells and Kras ${ }^{\mathrm{G} 12 \mathrm{D}}$ pancreatic epithelial cells , $\mathrm{p}<0.0001$. H)

606 Gene set enrichment analysis (GSEA) of dataset, described in (52) F).

607 Figure 3 RUNX1 inhibition induces a global redistribution of active chromatin and activates of 608 the proximal promotor region of the NOXA gene

609 A) Volcano Plot of differential expressed genes. MiaPaCa-2 cells were treated with vehicle

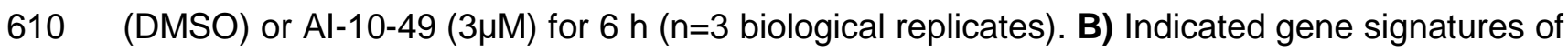
611 a GSEA from RNAseq data, described in A). GSEA, gene set enrichment analysis. C) Heatmap representing open chromatin peaks by omni-ATAC-seq analysis in DMSO and Al-10-49

613 treated cells $(3 \mu \mathrm{M}$ for $6 \mathrm{~h})$ and in parental and $R U N X 1^{k o}$ MiaPaCa-2 cells $(\mathrm{n}=2)$. D) Heatmap 614 representing H3K27ac peaks in MiaPaCa-2 cells treated for $6 \mathrm{~h}$ with vehicle (DMSO) or $3 \mu \mathrm{M}$ 615 Al-10-49 $(n=2)$. Venn diagram displays H3K27ac peaks in Al-10-49 and vehicle treated 616 MiaPaCa-2 cells. H3K27ac peak distribution over genomic features are displayed in a bar plot 617 diagram. E) Omni-ATACseq (DNA accessibility) ( $n=2)$, chromosome conformation capture 618 (4C, spatial chromatin organisation) $(n=1)$ and ChIPseq $(n=2)$ analysis for RUNX1, H3K27ac and CTCF in vehicle (DMSO)- or Al-10-49 treated MiaPaCa-2 cells. Arrows indicate either 620 RUNX1 binding and an interaction of the NOXA gene at downstream binding site 1 (dBS1). F) 621 ChIP-qPCR analysis at the promoter and the downstream binding site 1 (dBS1) for RUNX1, H3K27ac and IgG in DMSO- or Al-10-49 treated MiaPaCa-2 cells ( $n=3)$. G) Heatmap of Hi-C data from Panc1 cells generated by Dekker laboratory (36), accessed via http://3dgenome.fsm.northwestern.edu/ displaying spatial chromatin organization. H) Relative NOXA mRNA expression in indicated clones of MiaPaCa-2 cells. The dBS1 binding site was excised using CRISPR/Cas9. Cells were treated for $6 \mathrm{~h}$ with $3 \mu \mathrm{M}$ of Al-10-49. I) Western blot 627 of NOXA $6 \mathrm{~h}$ upon treatment with $3 \mu \mathrm{M} \mathrm{Al}-10-49$ in indicated clones of MiaPaCa-2 cells. Actin 628 served as loading control. J) Schematic of RUNX1 mediated repression of the NOXA gene.

629 Figure 4 Tumor growth is blocked by RUNX1 inhibition in vivo and in patient derived organoids.

630 A) Mice were treated with $200 \mathrm{mg} / \mathrm{kg}$ Al-10-49 intra peritoneal daily for 9 days. Treatment 631 started (d1) when tumors reached a volume of $0.2 \mathrm{~cm}^{3}$. B) Tumor size was measured over 
632 time in parental and $N O X A^{k o}$ xenografts. Al-10-49 treated mice showed a significant tumor 633 growth inhibition ( $n=5$ mice in each group). ${ }^{* *} p<0.01,{ }^{* * *} p<0.001$ (Student's t-Test) C) IHC of 634 parental xenografts. Left panel: Representative pictures of tumors from Al-10-49 and vehicle 635 treated mice. Displayed are full scans of the tumors (bar: $2 \mathrm{~mm}$ ); detailed pictures of H\&E 636 stained and IHC for Ki67 and cleaved caspase 3 (CC3, bar: 100 $\mu \mathrm{m})$. Right panel: 637 Quantification of Ki67 and CC3 IHC staining of Al-10-49 treated tumor xenografts using the 638 Aperio positive pixel method. ${ }^{*} \mathrm{p}<0.055,{ }^{* *} \mathrm{p}<0.01$ (t-test). D) IHC of $N O X A^{k o}$ xenografts as 639 indicated in C). E) RNA-seq data of 7 patient derived organoids (PDOs) were analyzed for 640 NOXA expression. NOXA mRNA expression $>75 \%=N O X A^{\text {high; }}$, NOXA mRNA expression $<$ $64125 \%=N O X A^{\text {low }}$. F) GSEA of RNA-seq data of PDOs. Hallmark apoptosis signature in the $642 N O X A^{\text {high }}$ subtype. Nominal p-value $<0.001$. FDR-q value is depicted. GSEA, gene set 643 enrichment analysis. G) Dose-response treatment of PDOs viability measured after 72h upon $644 \mathrm{Al}-10-49$ treatment with CellTiterGlo ${ }^{\circledR}$.

\section{Supplements}

646 SFigure 1 Classical BH3 only mRNA expression and survival analysis in PDAC

647 A) mRNA expression of BH3-only differentially expressed in PDAC subtypes classified in 648 squamous and other. Fisher's exact test, ${ }^{*} p<0.01,{ }^{* *} p<0.001,{ }^{* *} p<0.0001$. B) Simple 649 Cox Regression models were constructed using the expression of all "classical BH3 family 650 members". Genes with p-values $<0.1$ were used to construct a multiple Cox regression model. 651 Out of all members, only NOXA significantly correlated with inferior survival $(\mathrm{HR}=1.9, \mathrm{p}<$ 0.001), while increasing expression of $B B C 3 / P U M A$ was associated with higher overall survival $653((\mathrm{HR}=0.73, \mathrm{p}=0.095) . \mathrm{C})$ Survival of PDAC patients with a low (lower quartile) and a high 654 (upper quartile) NOXA mRNA expression derived from curated TCGA datasets of PDAC 655 patients as described (13). Log rank test as indicated. D) Human PDAC cell line subtypes 656 according to Collisson et al. (8). QM: quasi-mesenchymal.

657 SFigure 2 CRISPR/Cas9 knockout and CRISPRa strategies and co-immunopreciptitation of 
A) Scheme of the human NOXA gene (parental and knockout) with indicated primer binding sites. B) Genotyping PCR of indicated human PDAC cells to screen for a NOXA knockout. C) Scheme of the murine NOXA gene (parental and knockout) with indicated primer binding sites. D) Genotyping PCR of indicated murine PDAC cells to screen for a NOXA knockout. E) Coimmunoprecipitation of RUNX1 and CBF $\beta$ in MiaPaCa-2 cells. Cells were treated for $6 \mathrm{~h}$ with $3 \mu \mathrm{M}$ of Al-10-49 or DMSO as vehicle control. Either a RUNX1 or a CBF $\beta$ antibody was used representation of dCas9-VP64-MS2-HSF1 driven NOXA overexpression. One sgRNA drives dCas9 towards the NOXA promoter region to induce its expression. G) Schematic representation of RUNX gene family knockout. For RUNX1 knockout, 2 sgRNAs were designed to excise the gene region from exon 3 to exon 4. For RUNX2 knockout, 2 sgRNAs were designed to target excision of beginning of exon 8 until stop codon. For RUNX3 knockout, 2 sgRNAs target upstream exon 1 and end of exon 2 .

A) Western blot analysis of indicated BCL2 family members and cleaved PARP upon 6h and 24h treatment with $3 \mu \mathrm{M}$ of Al-10-49 in MiaPaCa-2 and PSN1 cells. Actin served as loading control. B) Protein expression analysis of MCL1, p53, BCL-xL, BIM and BAX upon 24h and 72h treatment with $3 \mu \mathrm{M}$ of $\mathrm{Al}-10-49$ and the pan-caspase inhibitor zVAD-FMK in parental and NOXA ${ }^{\text {ko }}$ MiaPaCa-2 cells. GAPDH served as loading control. C) p53 Western Blot of indicated 678 murine PDAC cell lines to determine basal $p 53$ expression of $n=3$ biological replicates. Actin 679 served as loading control. D) Relative Noxa mRNA expression in murine PDAC cells harboring wild type p53 (PPT-5123), mutant p53 (PPT-5436) and deleted p53 (PPT-6554 and PPT-W22). 681 Cells were treated for $6 \mathrm{~h}$ with $12.5 \mu \mathrm{g} / \mathrm{ml}$ Etoposide, $3 \mu \mathrm{M} \mathrm{Al}-10-49$ and DMSO as vehicle control. Actin served as housekeeping gene. E) Western blot of RUNX1 and p63 $\alpha$ upon 6, 24 and $48 \mathrm{~h}$ treatment with $3 \mu \mathrm{M}$ of $\mathrm{Al}-10-49$ in parental and $N O X A^{k o} \mathrm{MiaPaCa}-2$ cells and parental 684 PSN1 cells. GAPDH served as loading control. F) AnnexinV/PI FACS of parental and NOXA 685 MiaPaCa-2 cells upon 24h treatment with $3 \mu \mathrm{M}$ Al-10-49 and 50 $\mu \mathrm{M}$ zVAD-FMK. Upper panel: 
AnnexinV+/PI' fraction. Lower panel: Annexin $\mathrm{V}^{+} / \mathrm{PI}^{+}$fraction. G) Western blot of NOXA protein in MiaPaCa-2 cells. Vinculin served as loading control. H) Representative image of clonogenic assay in MiaPaCa-2 parental and NOXA-CRISPR-dCas9-VP64-MS2-HSF1-mediated activation (NOXA-CRISPRa). Cells were treated for 3 weeks with vehicle (DMSO) or $400 \mathrm{nM}$ Al-10-49. $n=4$; all biological replicates were performed as technical triplicates. I) Quantification of clonogenic assay in parental and NOXA-CRISPRa. Each treatment was quantified and normalized against its DMSO control. Depicted is the number of colonies in \% per treatment compared to vehicle. $P$ value of ANOVA test ${ }^{* *} p<0.001$

SFigure 4 RUNX1 is upregulated in pancreatic cancer and suppresses apoptosis via NOXA repression

A) Relative RUNX1 and NOXA expression in MiaPaCa-2, Panc-1 and AsPC1 cells upon treatment with a pool of specific siRNAs targeting RUNX1. Cells were treated for $72 \mathrm{~h}$ with either scrambled siRNAs (Ctrl) or RUNX1 specific siRNAs. Actin was used as housekeeping gene $(\Delta \Delta \mathrm{Ct})$. B) GI50 values generated in viability assays using CellTiterGlo in parental, RUNX1ko and cells, determined 72h after Al-10-49 treatment. In addition cells were treated 48h with siRNA (Ctrl and RUNX1) and treated for additional $72 \mathrm{~h}$ with Al-10-49. C) RUNX1 mRNA expression of patient samples from normal pancreas and pancreatic cancer GEO accession no. GSE15471, GSE16515 D) Pearson correlation (incl. linear regression with 95\% confidence bands) of PDAC patient samples from the squamous subtype (6) and from microdissected PDAC patient samples (8). E) Western blot of RUNX1 and NOXA in parental and $R U N X 1^{k o}$ MiaPaCa-2 cells. $R U N X 1^{k o}$ cells were stably transduced with either a control shRNA or a NOXA specific shRNA. Actin served as loading control. F) Annexin V FACS 708 analysis of cells as indicated in E).

SFigure 5 Quality assessment of H3K27ac ChIP-seq.

710 A) Fingerprint plot of all replicates including the input controls. Samples with uniform 711 distribution across the genome (e.g. input controls) should plot across the diagonal line, while 712 samples with enrichment across small genomic regions show a steep rise towards the end of 
713 the plot. B) Correlation plot calculated using the Spearman coefficient quantified by the 714 horizontal bar at the bottom of the plot. C) Average signal profile across peaks calculated with 715 macs2 callpeak. The profile was generated with ChIPQC.

716 SFigure 6 RUNX1 suppresses NOXA via the dBS1 downstream enhancer in PDAC cells

717 A) Chromosome conformation capture (4C, spatial chromatin organisation) and ChIPseq 718 analysis for RUNX1 in indicated cell lines. MiaPaCa-2 cells were treated with vehicle (DMSO)719 or $3 \mu \mathrm{M} \mathrm{Al-10-49}$ for 6h. RUNX1 ChIPseq of MCF10A breast epithelial cells (GSE121370) and K562 CML cells (GSE96253) are displayed. The NOXA downstream binding site 1 (dBS1) is depicted. B) Heatmap of Hi-C data from K562 and MCF10A cells accessed via http://3dgenome.fsm.northwestern.edu/ displaying spatial chromatin organization. C) Relative NOXA mRNA expression in MiaPaCa-2 and PSN1 cells. Cells were treated with $4 \mu \mathrm{M}$

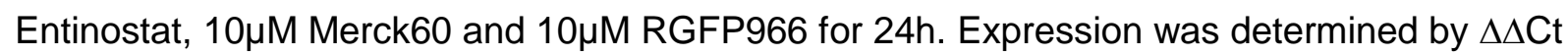
method. Actin served as housekeeping control. D) ChIP-qPCR analysis at the NOXA promoter for H3K27ac and IgG in DMSO- or Merck60 treated MiaPaCa-2 cells. E) Western Blot of HDAC1, HDAC2 and HDAC3 in murine PDAC cells harboring a dual recombination system. $600 \mathrm{nM}$ 4-Hydroxytamoxinfen (4-OHT) treatment induced CreERT2 shuttling and excision of either Hdac1 (PPT-F3641) or Hdac2 (PPT-F1648). Actin served as loading control. F) Relative Noxa mRNA expression in murine PPT-F3641 and PPT-F1648 cells. Cells were treated with $600 \mathrm{nM} 4-\mathrm{OHT}$ for $48 \mathrm{~h}$. Actin served as housekeeping control. G) Sequence analysis using ConTra v3 to identify conserved RUNX1 consensus sequences (as indicated) in the dBS1 region. H) Upper Panel: Scheme of the human dBS1 region (parental and knockout) with indicated primer binding sites. Lower Panel: Genotyping PCR of MiaPaCa-2 cells to screen for a dBS1 knockout. wt: wildtype; ko: knockout.

STable 1 Mean fold change of drug hits from drug screening experiment 
739 STable 4 PDO Gene Expression Matrix

740 STable 5 Primer sequences, sgRNAs, Antibodies, murine cell lines 
Fiaure 1 bioRxiv preprint doi: https://doi.org/10.1101/2021.10.21.465266; this version posted October 21, 2021. The copyright holder for this preprint (which was not certified by peer review) is the author/funder. All rights reserved. No reuse allowed without permission.

A

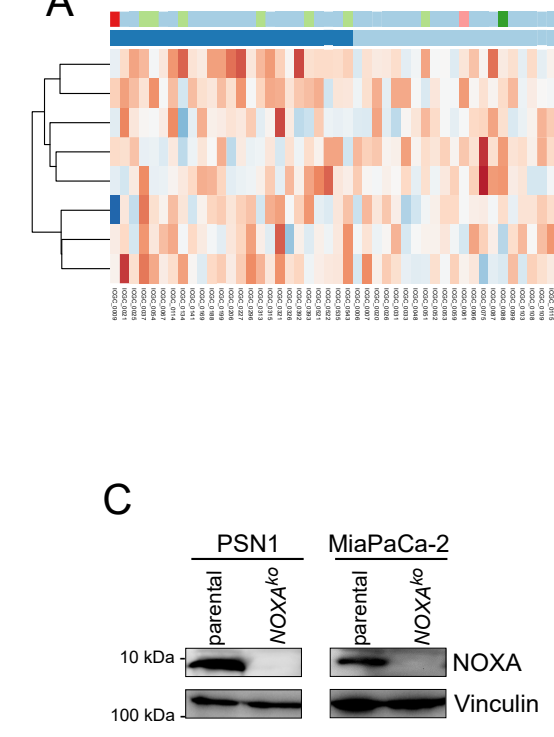

D

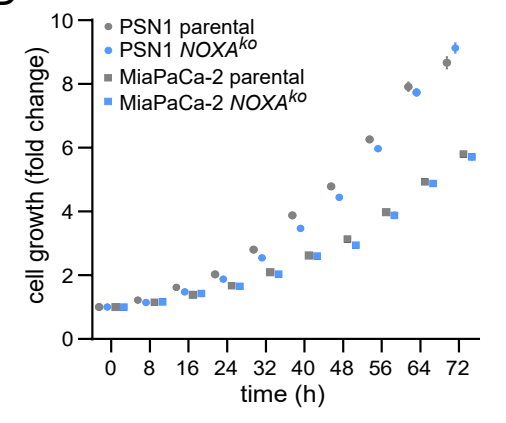

B ${ }^{100}$ 每 $\quad$ INOXA $^{\text {high }}(n=20)$

2 other

Equamous

0 Histology

1 PDAC

PDAC - signet ring

PDAC - undifferentiated

PDAC - adenosquamous

PDAC - mucinous non-cystic osteoclast like giant cells

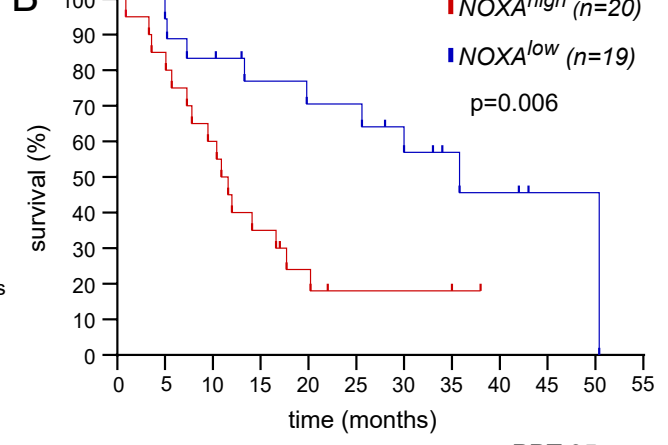

E PSN1 MiaPaCa-2

PPT-06

PPT-95

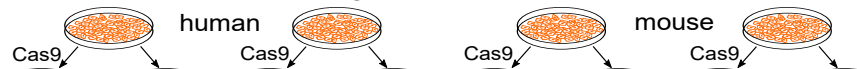

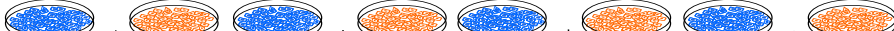

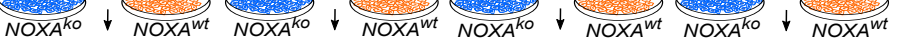
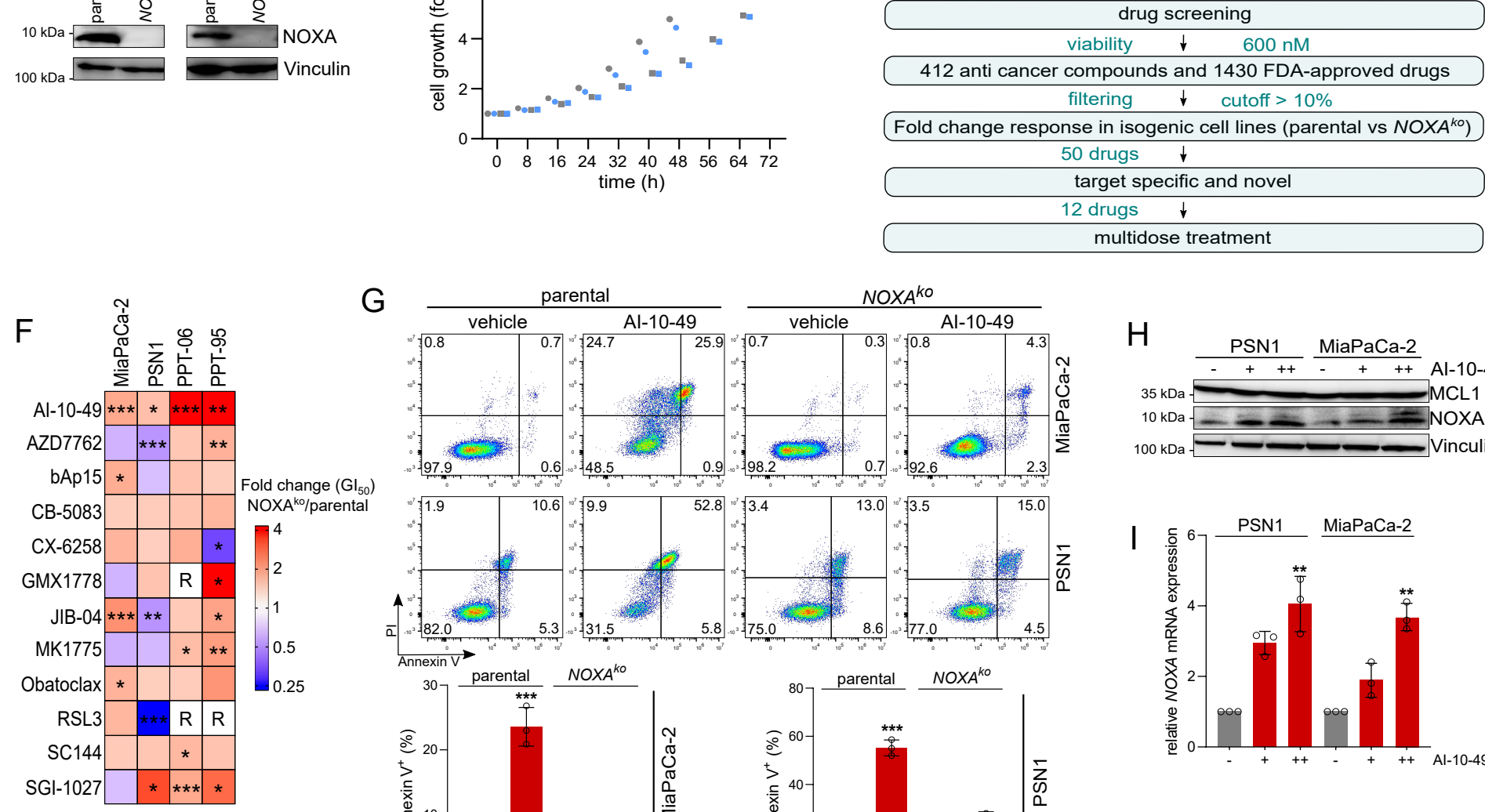

G
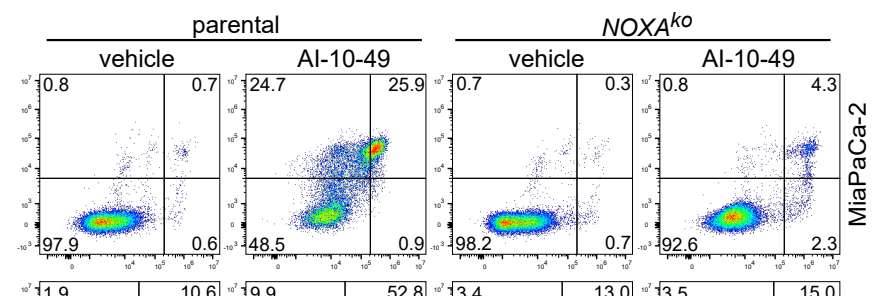

$\mathrm{H}$

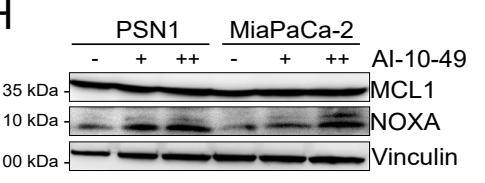

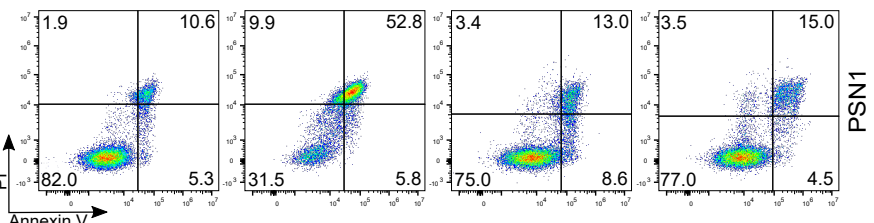
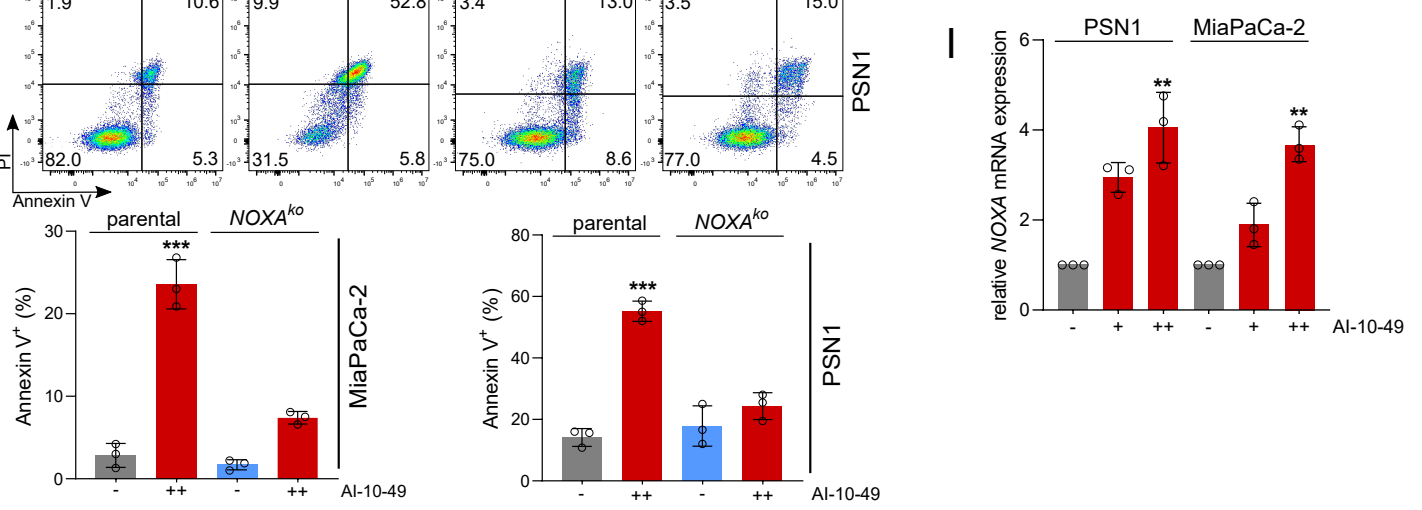
FioRxiv preprint doi: https://doi.org/10.1101/2021.10.21.465266; this version posted October 21, 2021. The copyright holder for this Figure 2 preprint (which was not certified by peer review) is the author/funder. All rights reserved. No reuse allowed without permission.
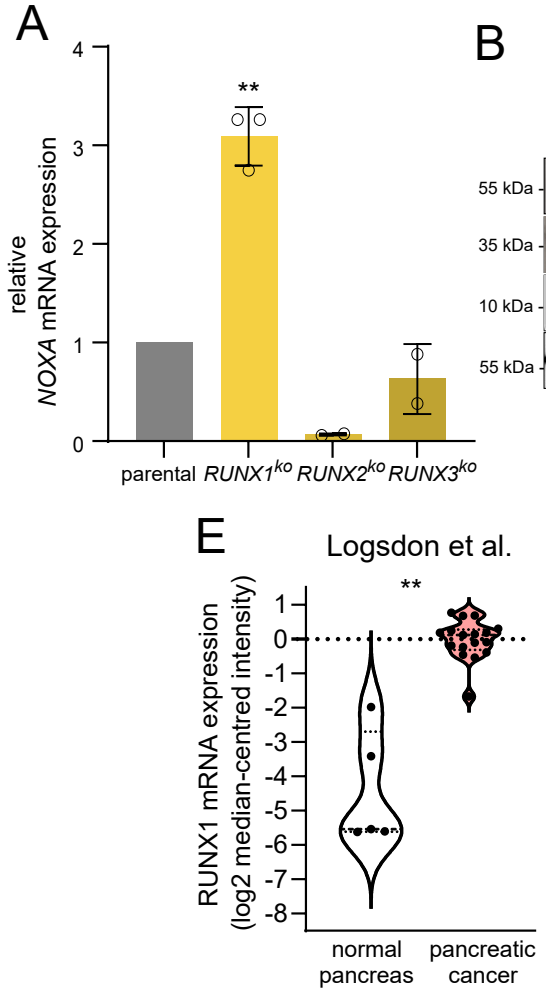

G

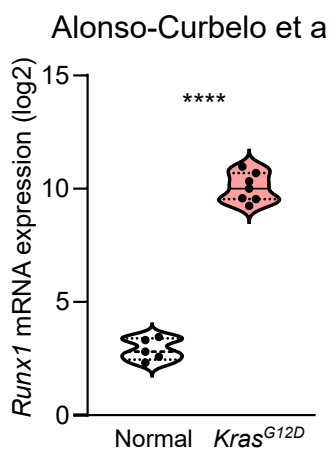

B

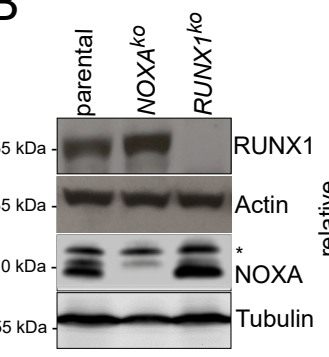

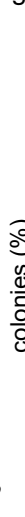

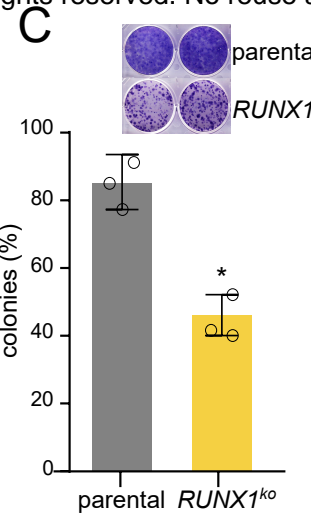

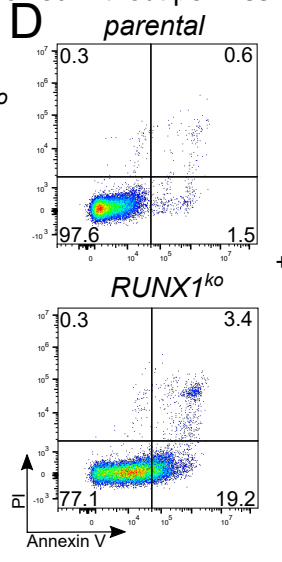

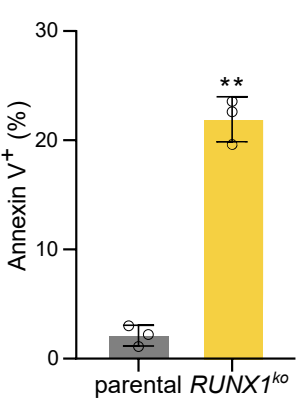

F
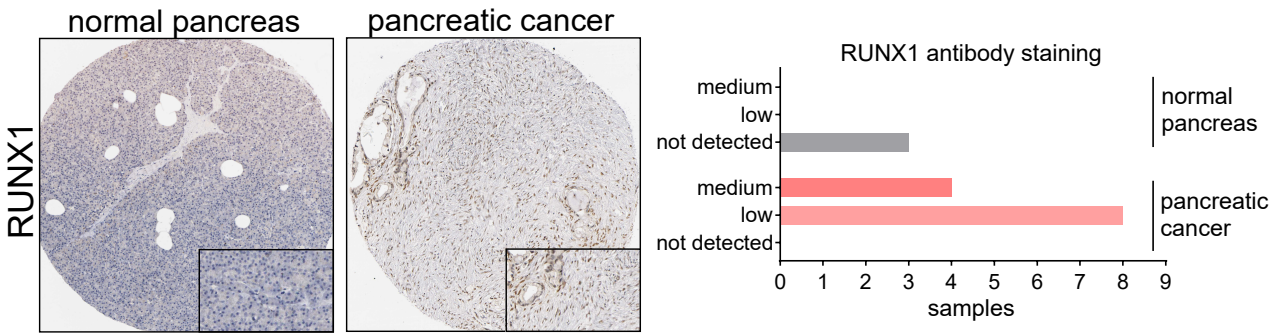

H Reactome: RUNX1 interacts with cofactors whose precise effect on RUNX1 targets is not known

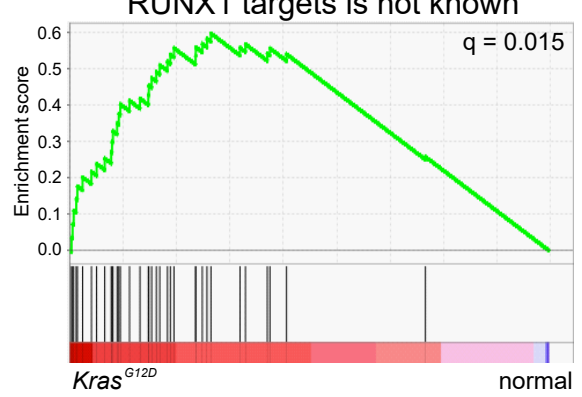

Reactome:

Transcriptional regulation by RUNX1

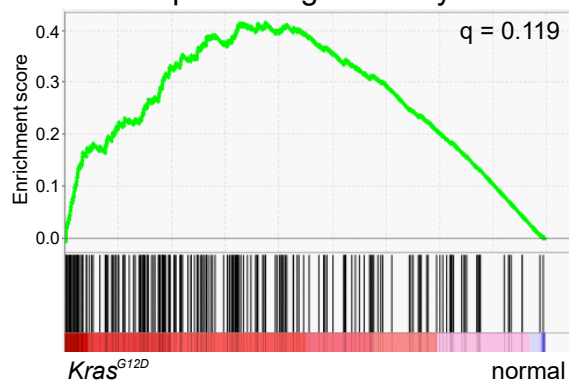


Fiqure 3 bioRxiv preprint doi: https://doi.org/10.1101/2021.10.21.465266; this version posted October 21, 2021. The copyright holder for this preprint (which was not certified by peer review) is the author/funder. All rights reserved. No reus eallowed Akithbewt permission.

A

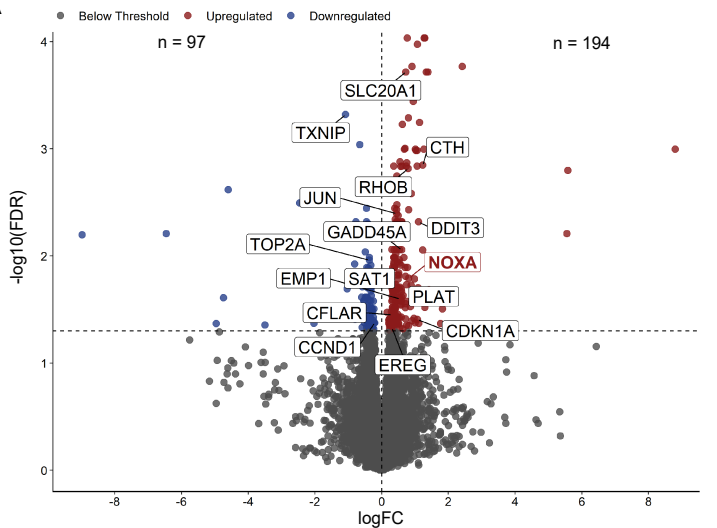

B

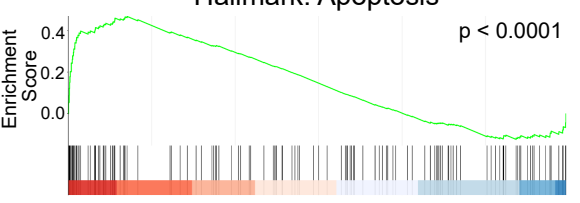

Reactome: Trancriptional regulation by RUNX1

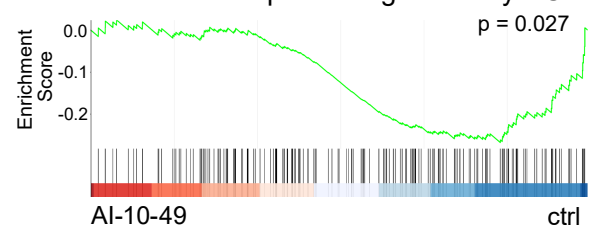

D

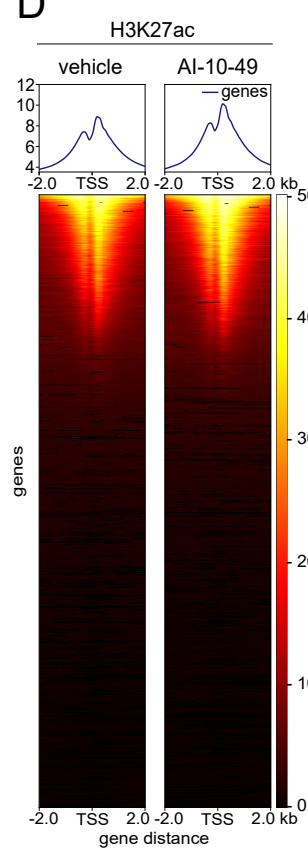

G

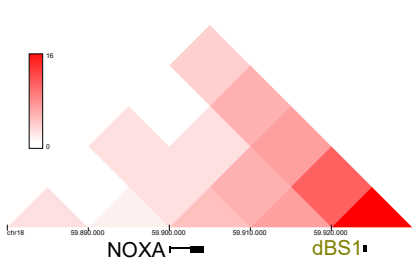

E

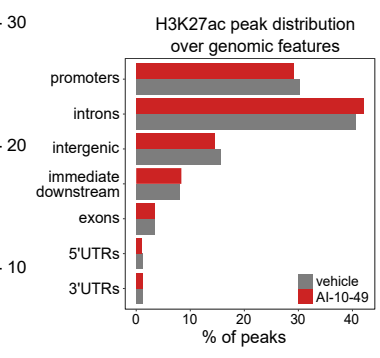

$\mathrm{H}$

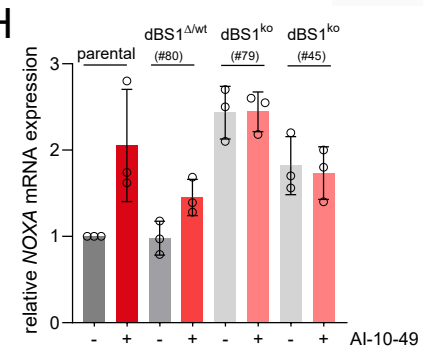

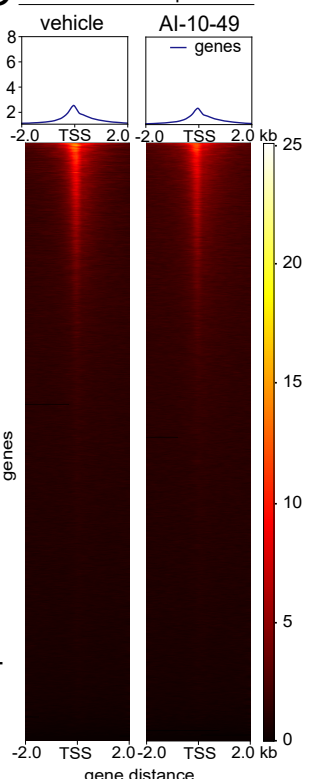

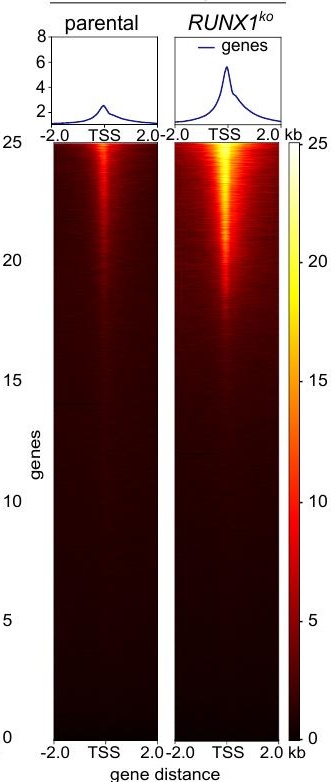

$\mathrm{F}$
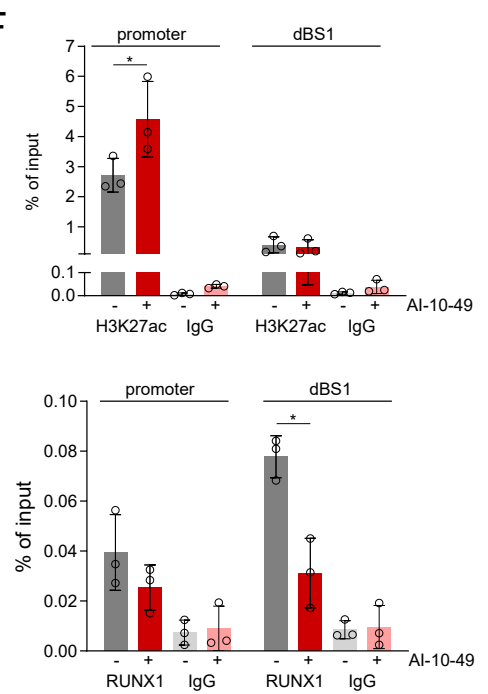

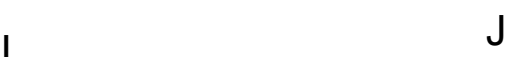

l-
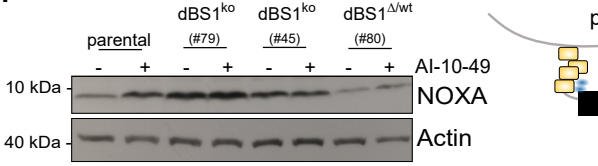

poised/inactive

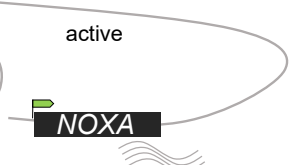

NOXA

$\square$ RUNX1 - H3K27ac - repressor $\frown$ mRNA 


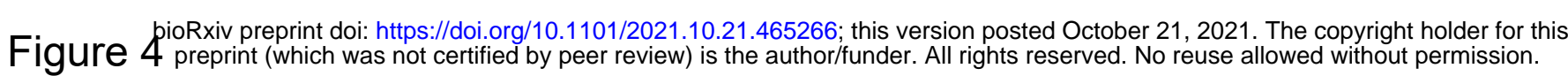

A

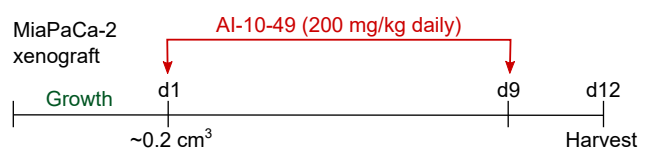

C

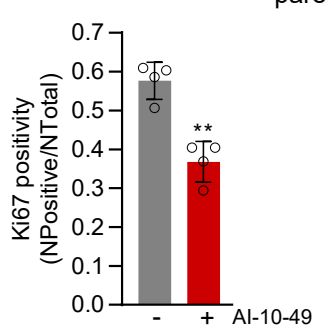

parental

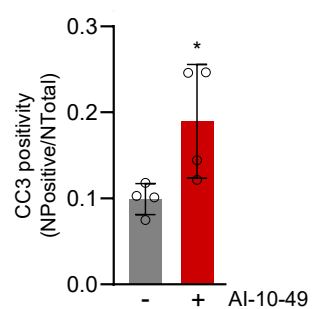

E

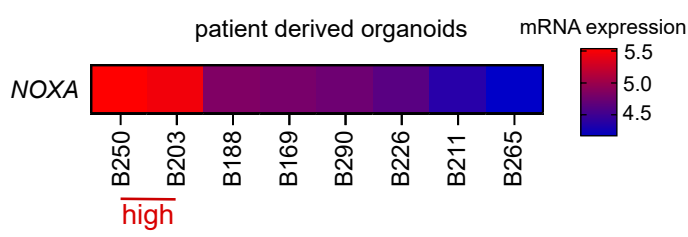

B

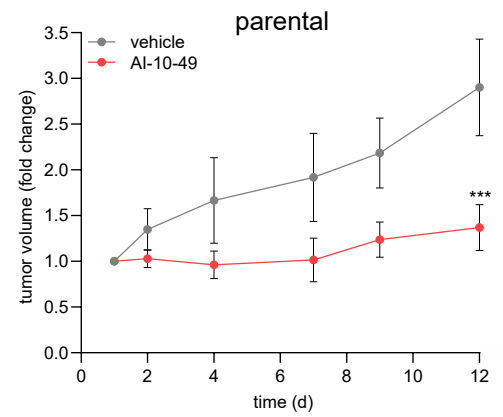

D

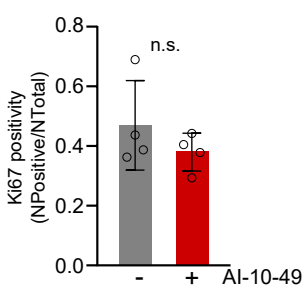

F

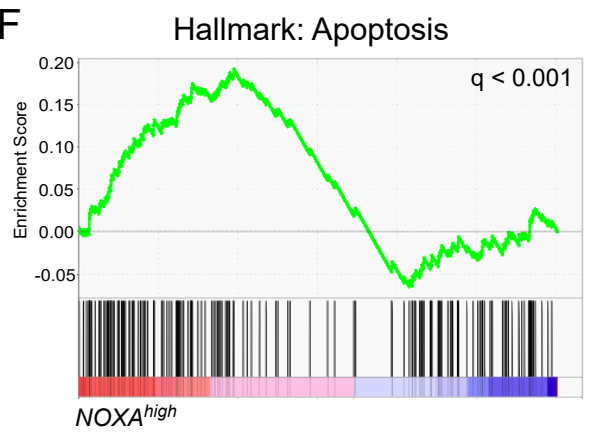

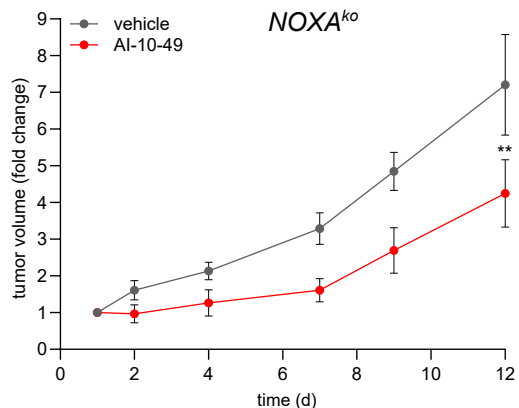

NOXAko

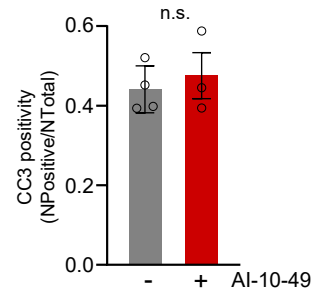

